\title{
Monolithic Carbon Spherogels as Free-standing Electrodes for Supercapacitors
}

\section{Supporting Information}

Miralem Salihovic, ${ }^{a}$ Philipp Schlee, ${ }^{\mathrm{b}}$ Servann Herou, ${ }^{\mathrm{b}}$ Maria-Magdalena Titirici, ${ }^{\mathrm{b}}$ Nicola Hüsing, ${ }^{\mathrm{a}}$ and Michael S. Elsaesser ${ }^{\mathrm{a}^{*}}$

a Chemistry and Physics of Materials, University of Salzburg, 5020 Salzburg, Austria

$b$ Department of Chemical Engineering, Imperial College London, South Kensington Campus, London, SW7 $2 A Z, U K$

* Corresponding author.

Tel: +43-662-8044-6262.E-mail: michael.elsaesser@sbg.ac.at 

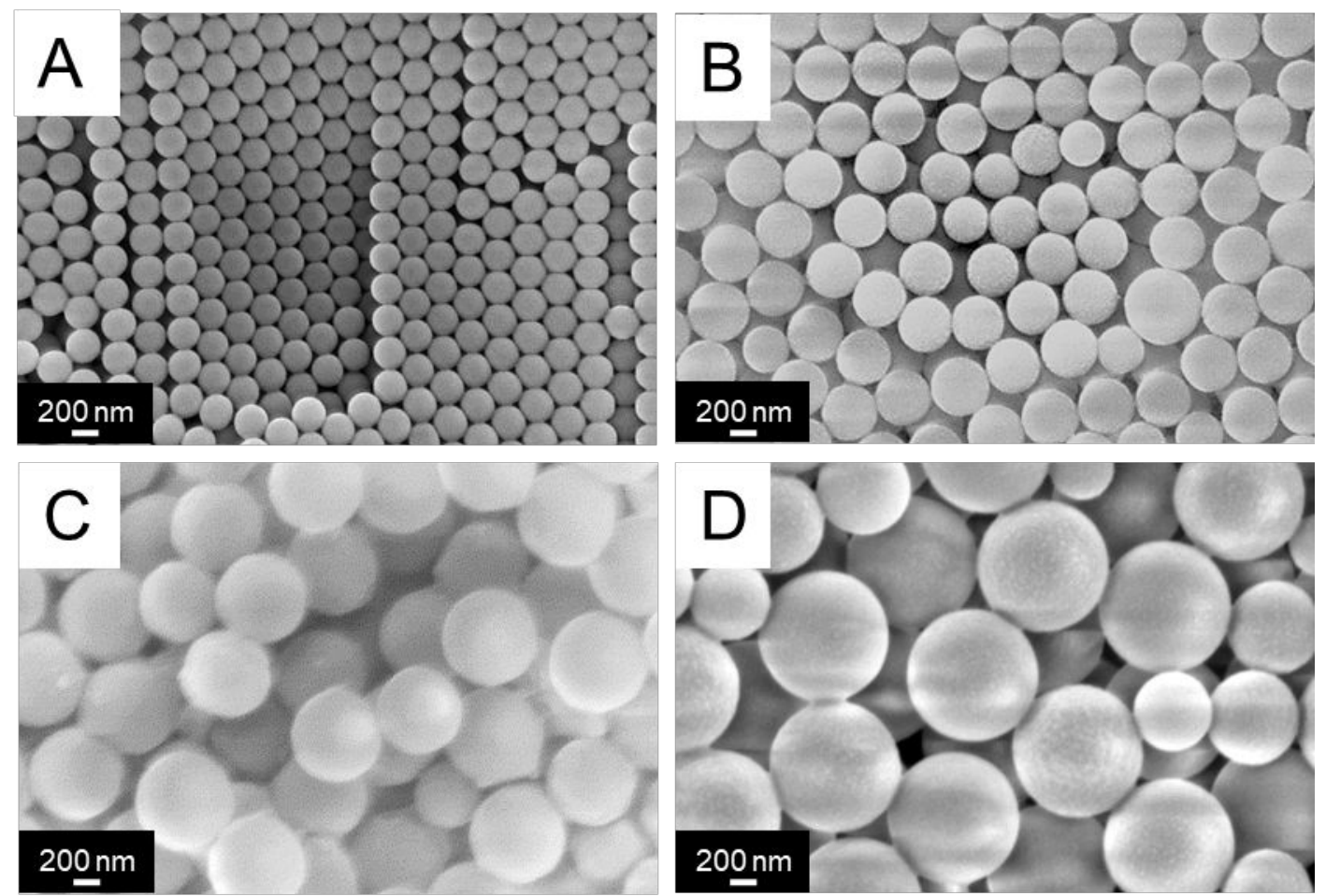

Figure S1: SEM micrographs of PS templating spheres with different diameters: (A) $277 \mathrm{~nm}$, (B) $455 \mathrm{~nm}$, (C) $736 \mathrm{~nm}$, (D) $907 \mathrm{~nm}$.
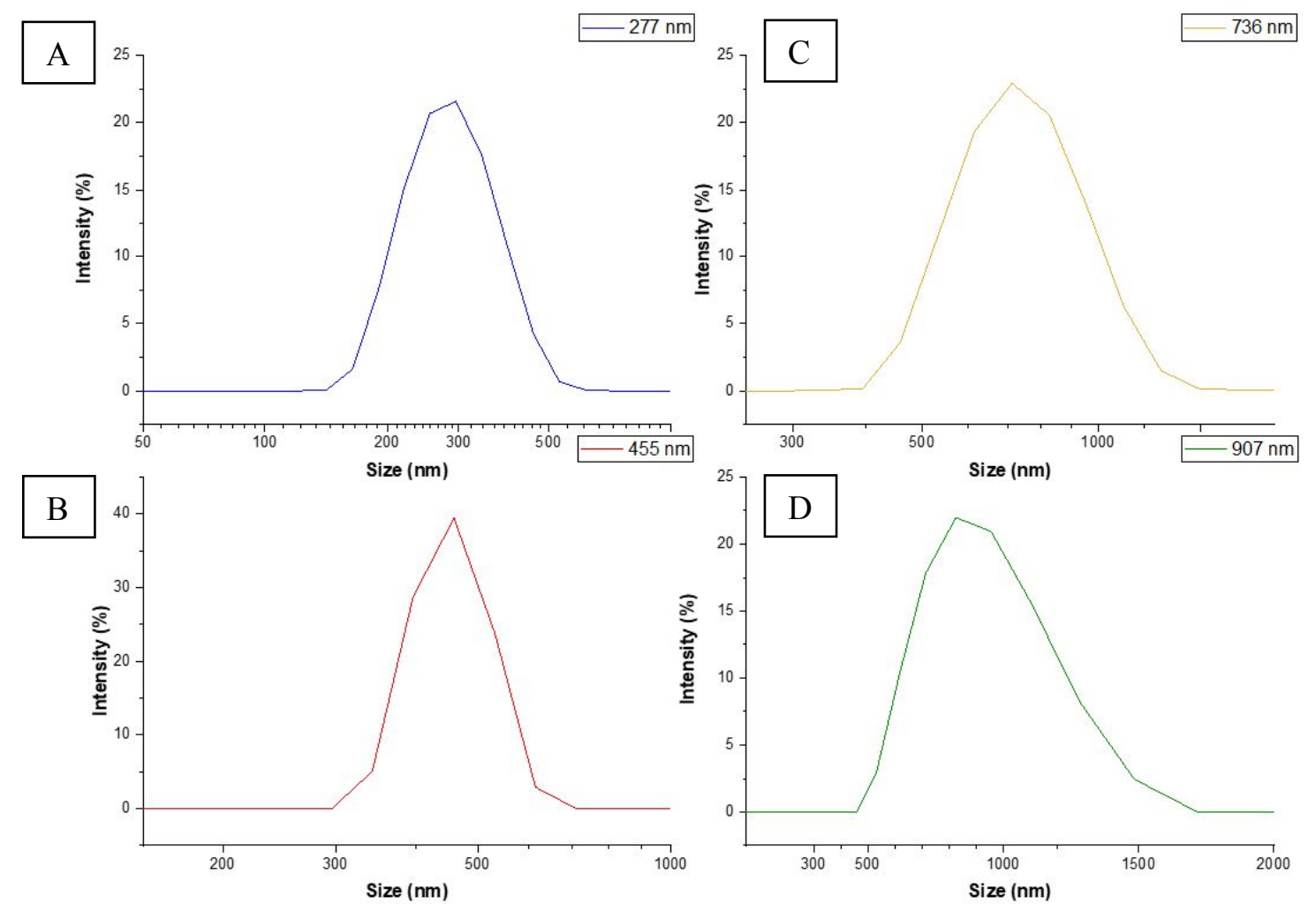

Figure S2: Particle size distribution of PS templating spheres in aqueous solution; (A) Z-average $=277 \mathrm{~nm}$ with PdI $=0.039$, (B) Z-average $=455 \mathrm{~nm}$ with PdI $=0.063$, (C) Z-average $=736 \mathrm{~nm}$ with PdI $=0.115$ and (D) Z-average $=907 \mathrm{~nm}$ with $\mathrm{PdI}=$ 0,176 . 


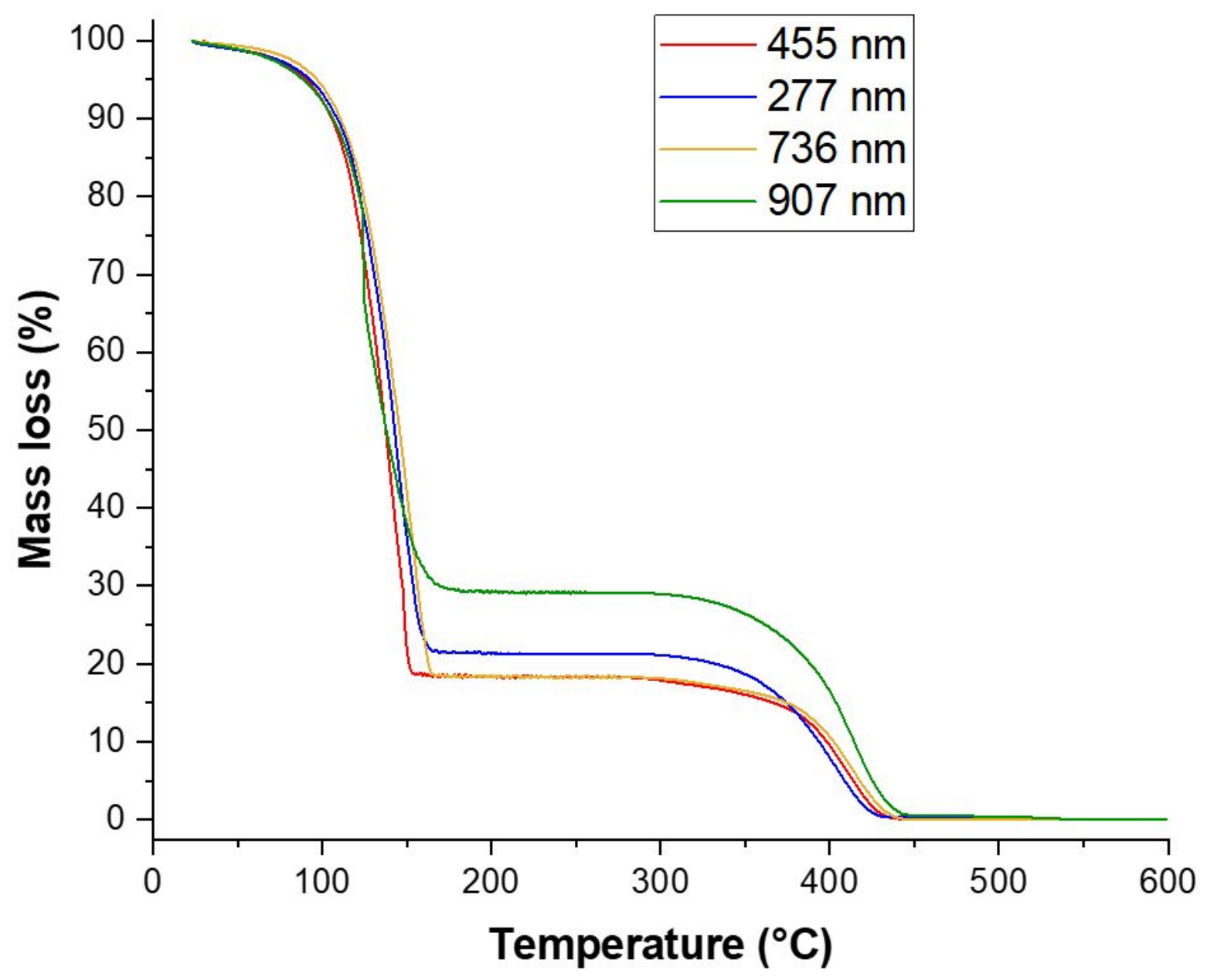

Figure S3: Thermogravimetric analysis of water evaporation $\left(<200^{\circ} \mathrm{C}\right)$ and decomposition $\left(<450^{\circ} \mathrm{C}\right)$ of colloidal PS solutions, blue curve: $277 \mathrm{~nm}$ particle diameter, red curve: $455 \mathrm{~nm}$ particle diameter, orange curve: $736 \mathrm{~nm}$ particle diameter, green curve: $907 \mathrm{~nm}$ particle diameter. 

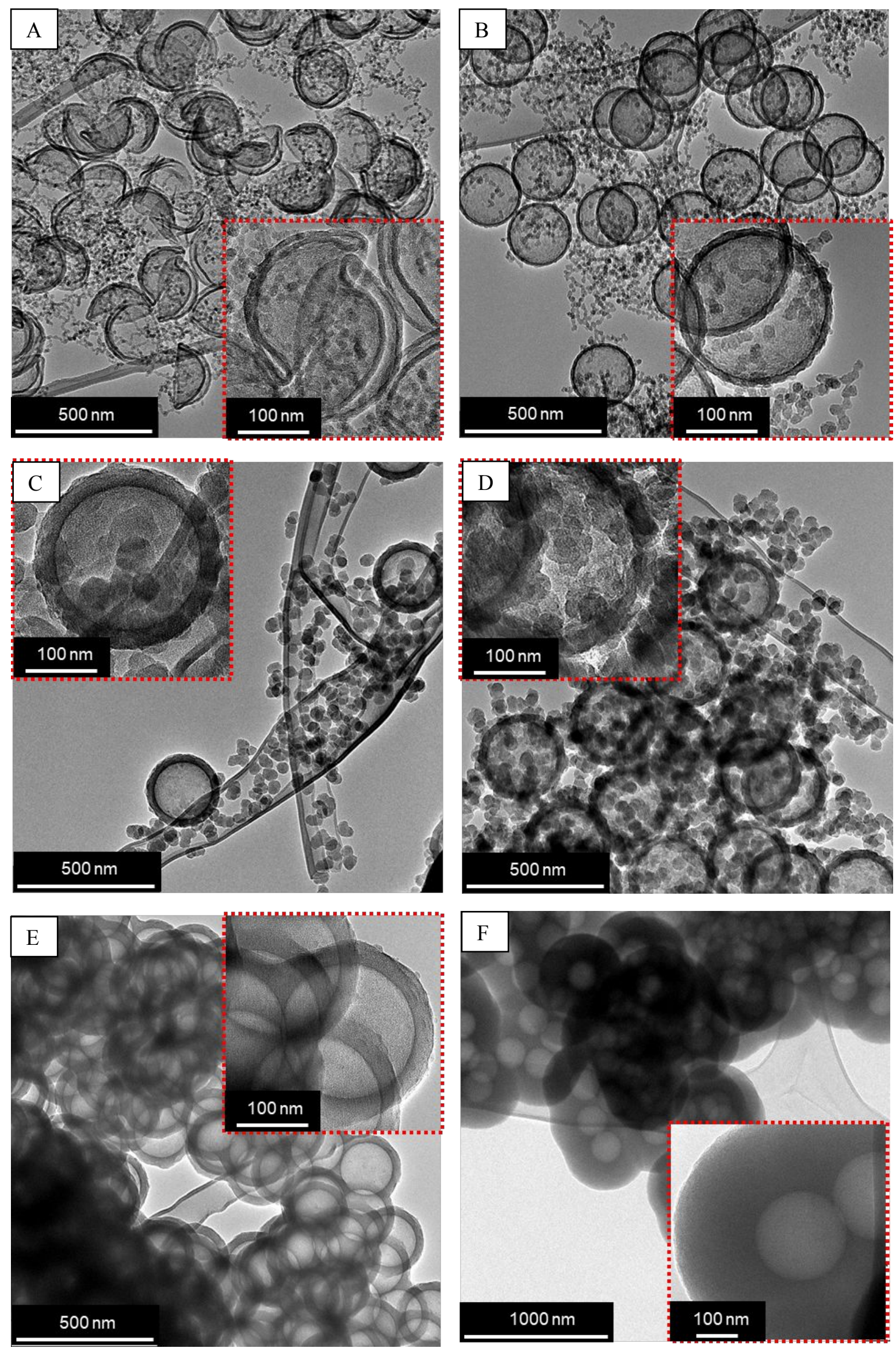

Figure S4: TEM micrographs of carbon spherogel samples prepared with different R/W molar ratios: 0.001 (A), 0.002 (B), $0.004(\mathrm{C}), 0.006(\mathrm{D}), 0.010(\mathrm{E}), 0.024$ (F) (PS template concentration constant at $1.5 \mathrm{wt} \%$ ). 

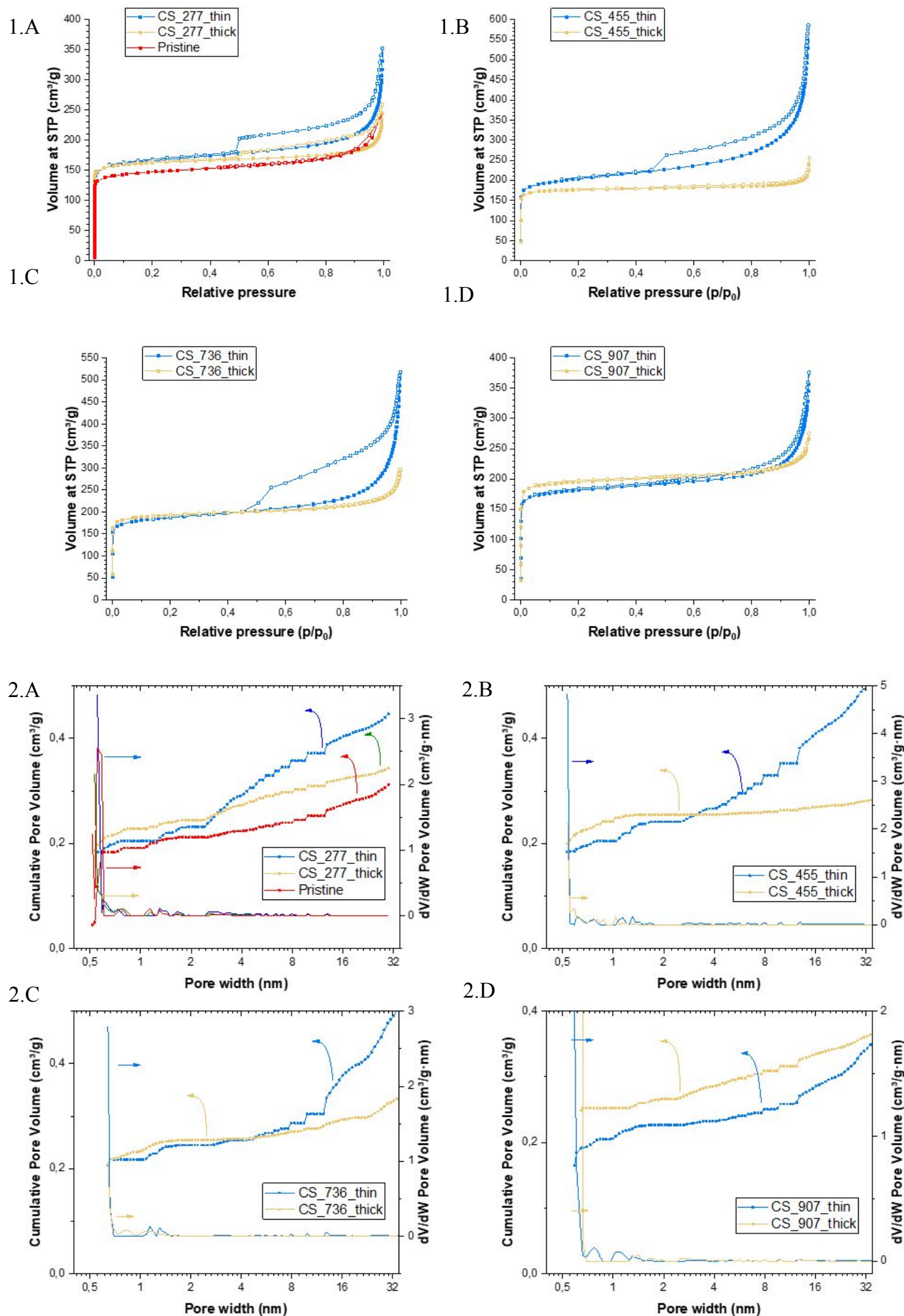

2.D

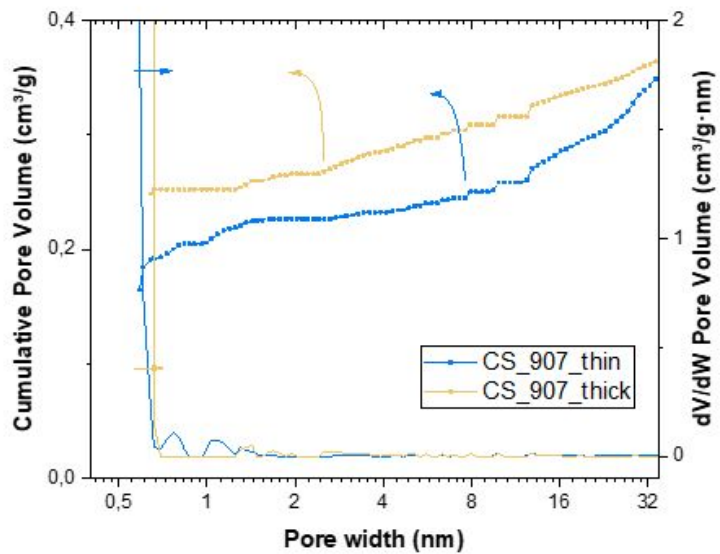

Figure S5: Nitrogen sorption isotherms (1) and pore width vs. cumulative pore volume and dV/dW pore volume distributions (2) measured at $-196^{\circ} \mathrm{C}$ (NLDFT carbon slit pore model) for carbon spherogel samples with different PS template diameters 
(A: $277 \mathrm{~nm}, \mathrm{~B}: 455 \mathrm{~nm}, \mathrm{C}: 736 \mathrm{~nm}, \mathrm{D}: 907 \mathrm{~nm}$ ) with thin wall (A: $14 \pm 1 \mathrm{~nm}, \mathrm{~B}: 22 \pm 2 \mathrm{~nm}, \mathrm{C}: 25 \pm 6 \mathrm{~nm}, \mathrm{D}: 34 \pm 6 \mathrm{~nm}$; blue curves), thick wall variants $(69 \pm 4 \mathrm{~nm}, 77 \pm 8 \mathrm{~nm}, 55 \pm 6 \mathrm{~nm}, 72 \pm 9 \mathrm{~nm}$; green curves) and a non-templated, pristine carbon aerogel sample (red curves).
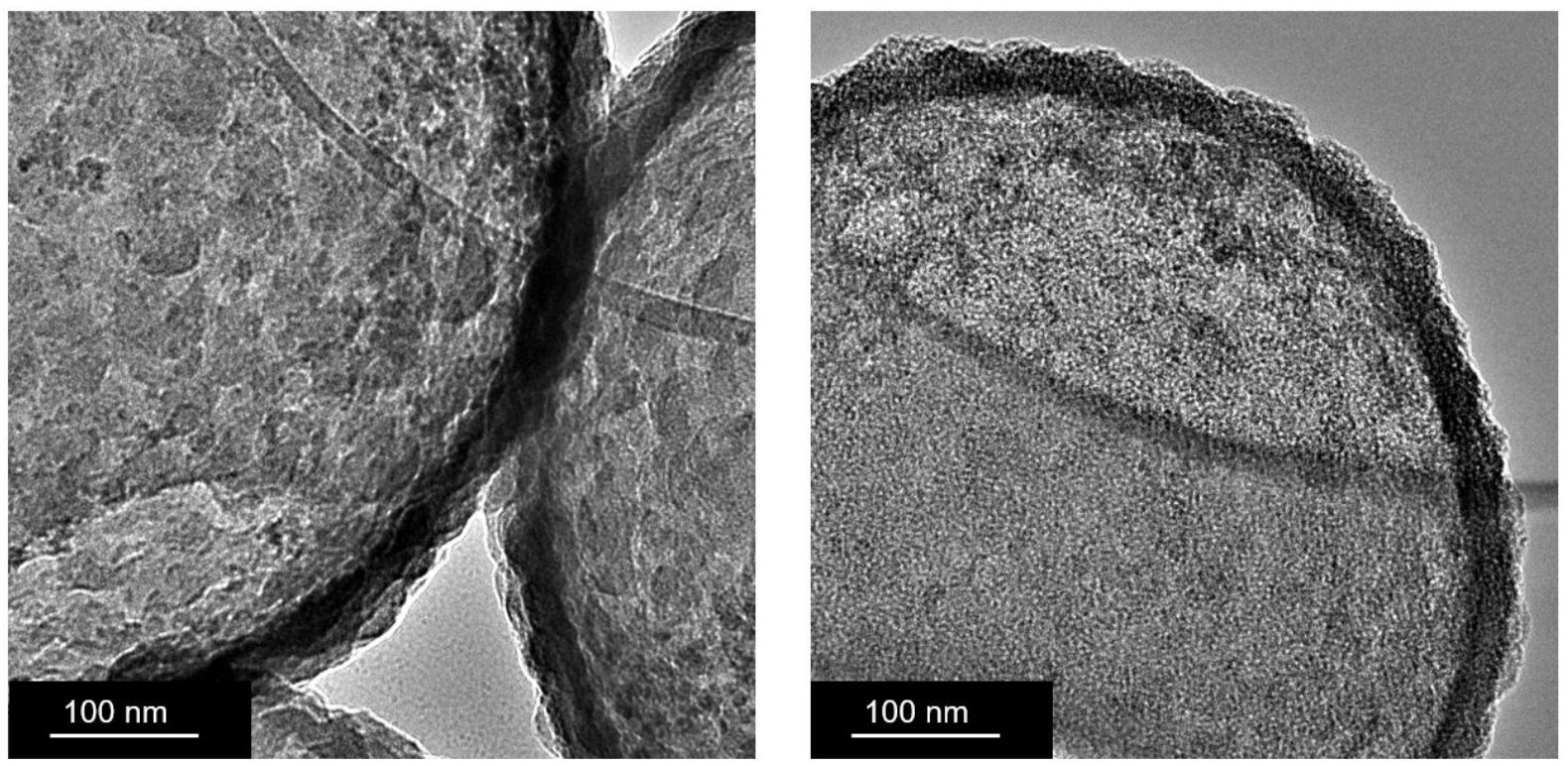

Figure S6: TEM micrograph of a carbon spherogel sample (CS_907_thin; 34 $\pm 6 \mathrm{~nm}$ wall thickness) before (left) and after (right) physical activation with carbon dioxide.
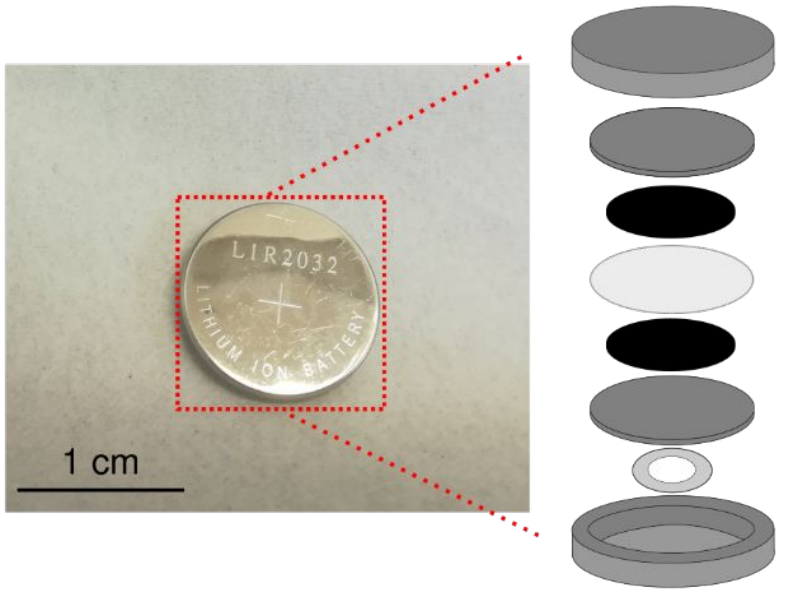

positive case
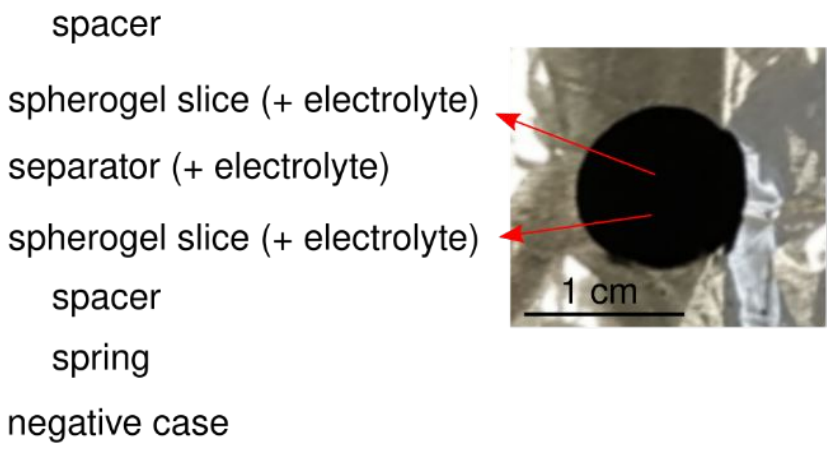

Figure S7: Schematic setup of the supercapacitor with photographs of assembled coin cells and a spherogel slice used as the electrode material 


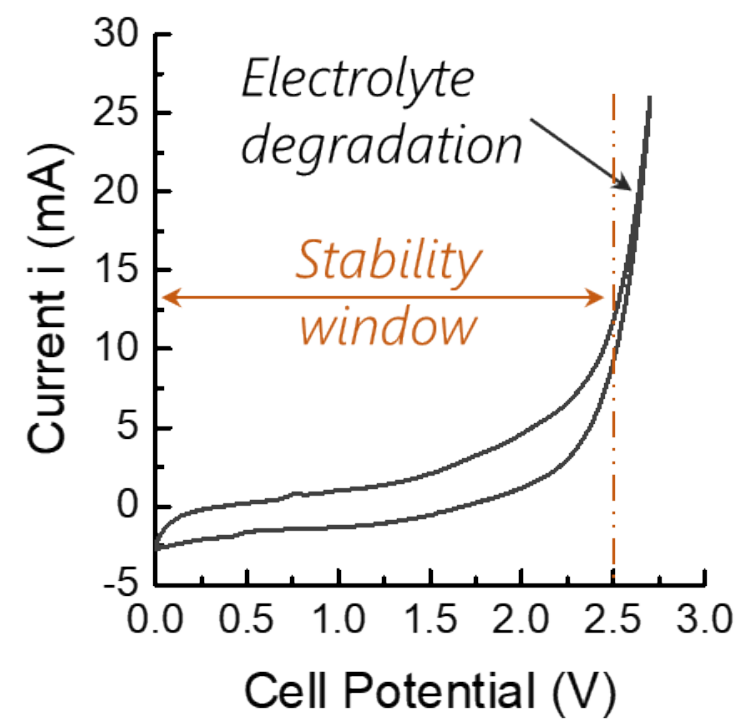

Figure S8: Cyclic voltammogram of a coin cell containing the neat electrolyte $1 \mathrm{M}$ TEA BF 4 in acetonitrile at a scan rate of 1 $\mathrm{mV} \mathrm{s}^{-1}$. The stability window was defined as $2.5 \mathrm{~V}$ as a degradation current peak is observed beyond $2.5 \mathrm{~V}$.
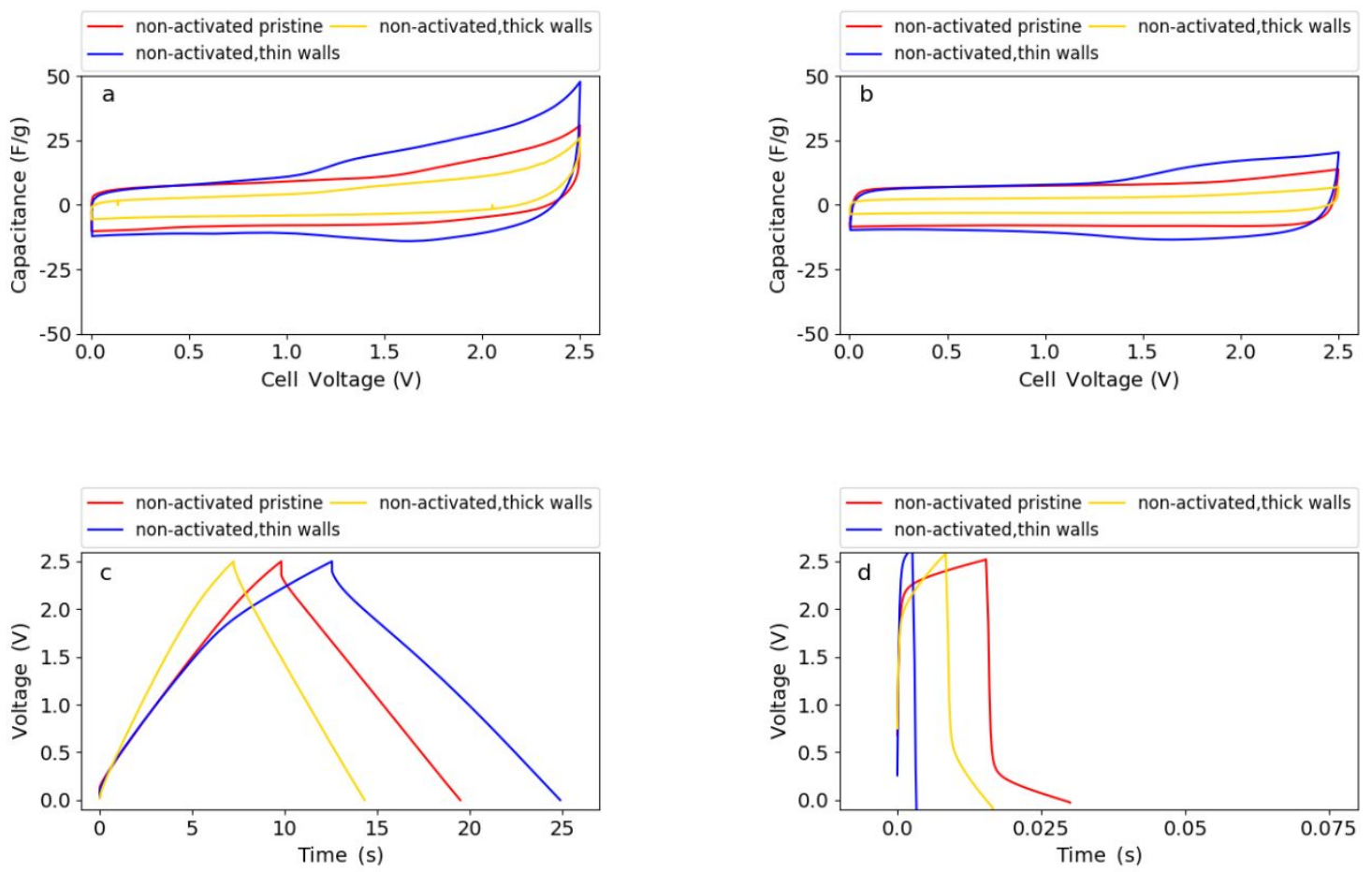

Figure S9: Voltammograms of the pristine and non-activated samples ( $277 \mathrm{~nm}$ spheres, thin and thick walls) at a) $\left.5 \mathrm{mV} \mathrm{s}^{-1}, \mathrm{~b}\right)$ $10 \mathrm{mV} \mathrm{s}^{-1}$, and galvanostatic charge-discharge traces of the non-activated pristine and non-activated spherogel samples $(277$ $\mathrm{nm}$ spheres, thin and thick walls) at c) $\left.0.1 \mathrm{~A} \mathrm{~g}^{-1}, \mathrm{~d}\right) 100 \mathrm{~A} \mathrm{~g}^{-1}$. 

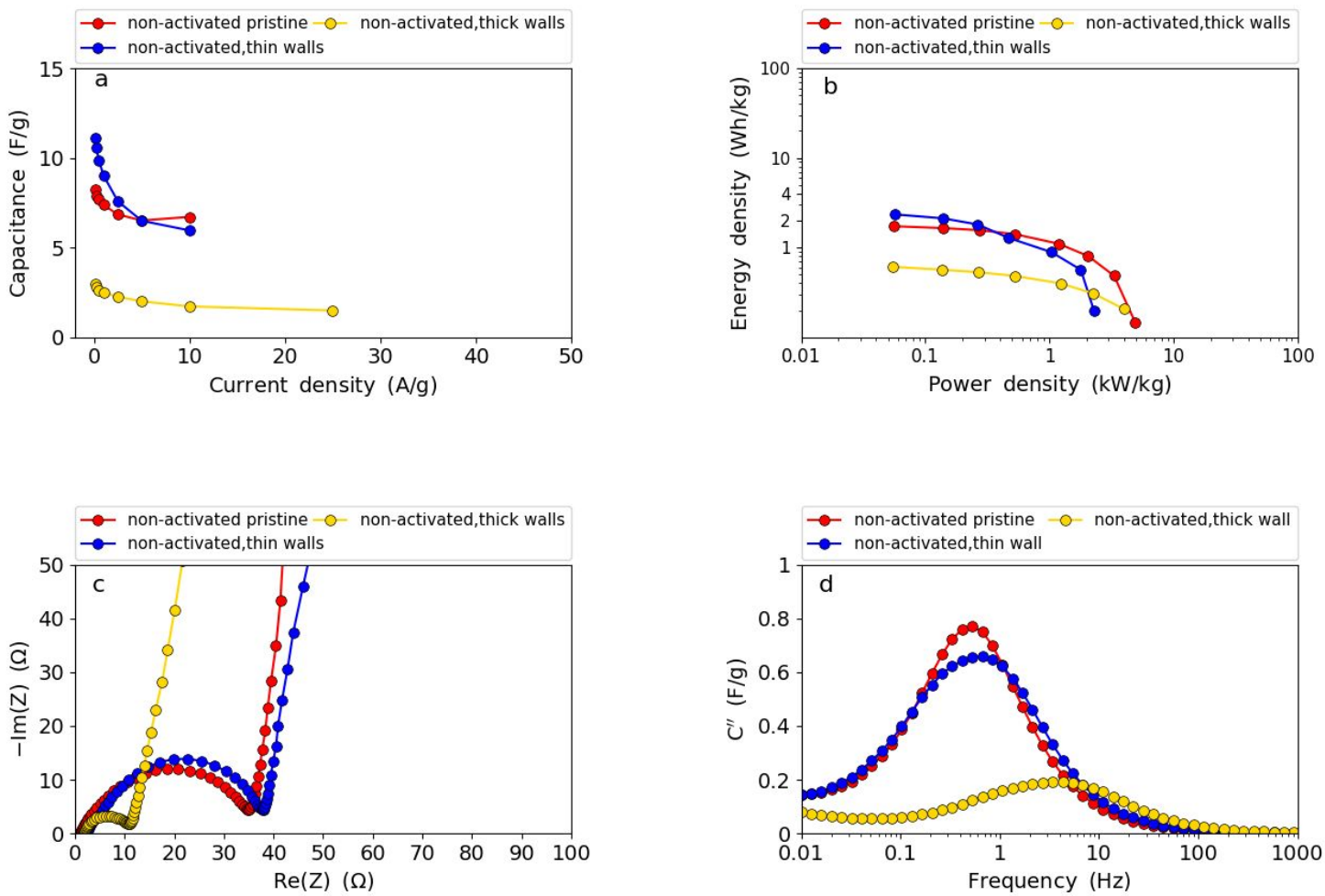

Figure S10: Capacitance retention of the pristine and non-activated samples ( $277 \mathrm{~nm}$ spheres, thin and thick walls) a), Ragone plot showing energy versus power density of the three samples b), Nyquist plot of the non-activated pristine and non-activated spherogel samples (277 nm spheres, thin and thick walls) c) and imaginary capacitance versus frequency d).
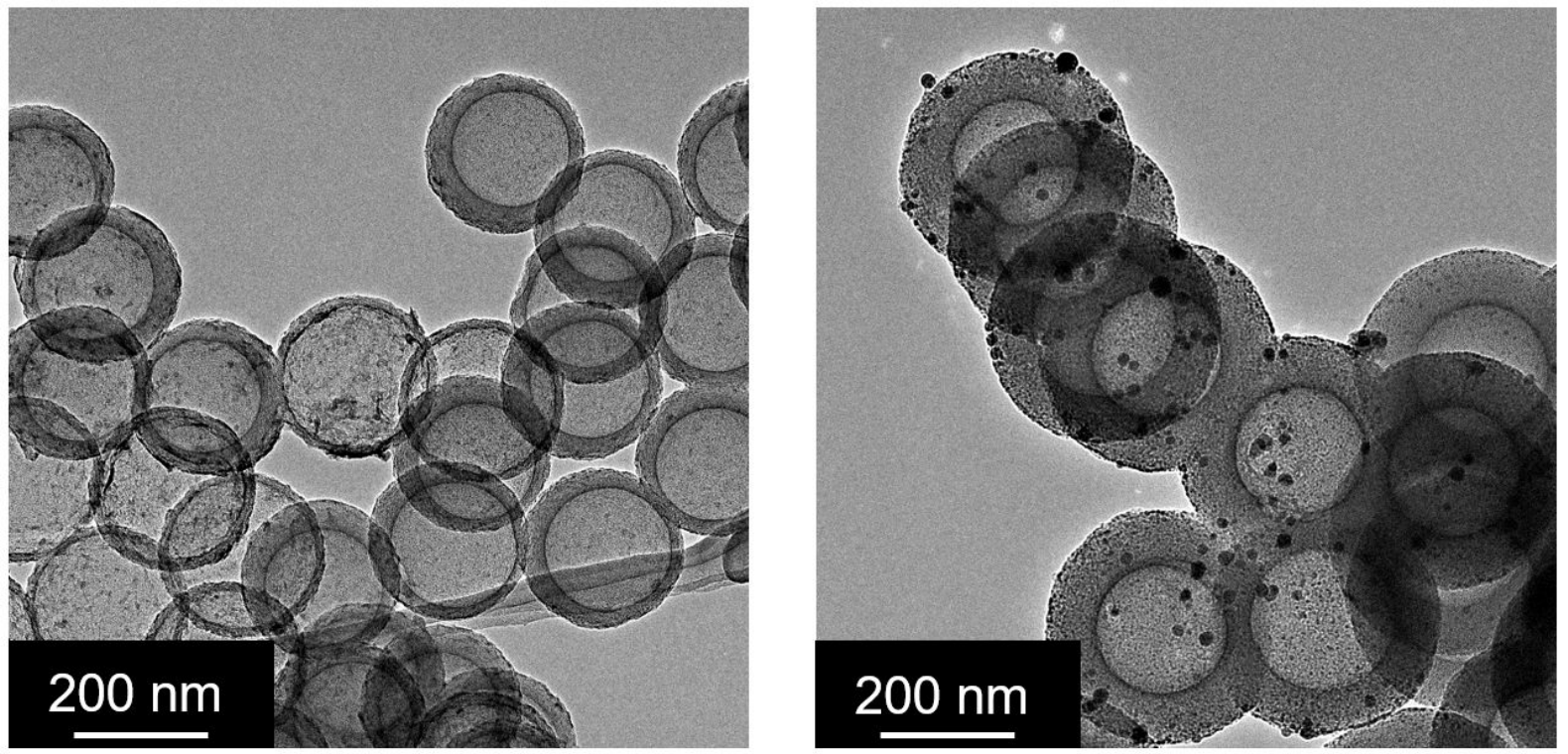

Figure S11: Post-mortem analysis. TEM micrograph of the templated, activated thin-walled $(14 \pm 1 \mathrm{~nm}$, left) and thick-walled $\left(69 \pm 4 \mathrm{~nm}\right.$, right) spherogel electrode with spheres sizes of $277 \mathrm{~nm}$, recorded after 6000 charge/discharge cycles at $10 \mathrm{~A} \mathrm{~g}^{-1}$ in $1 \mathrm{M} \mathrm{TEA} \mathrm{BF} 4$ in acetonitrile in a coin cell supercapacitor setup. 
Table S1: Mechanical and microstructural properties of carbon spherogels in correlation to the PS concentration in deionized water (at constant $\mathrm{R} / \mathrm{W}=0.008$ )

\begin{tabular}{|c|c|c|c|c|c|}
\hline PS (wt\%) & $\begin{array}{l}\text { Mechanical } \\
\text { behaviour }\end{array}$ & $\begin{array}{c}\text { Crack-free } \\
\text { mechanical cutting }\end{array}$ & Free-standing & $\begin{array}{c}\text { Spherogel } \\
\text { architecture }\end{array}$ & Wall thickness (nm) \\
\hline 0.38 & $\begin{array}{l}\text { Reversibly } \\
\text { compressible }\end{array}$ & Yes & No & No & $10 \pm 1$ \\
\hline 0.75 & $\begin{array}{l}\text { Reversibly } \\
\text { compressible }\end{array}$ & Yes & No & No & $13 \pm 1$ \\
\hline 1.50 & $\begin{array}{l}\text { Reversibly } \\
\text { compressible }\end{array}$ & Yes & No & No & $31 \pm 2$ \\
\hline 3.00 & $\begin{array}{l}\text { Reversibly } \\
\text { compressible }\end{array}$ & Yes & No & Yes & $32 \pm 4$ \\
\hline 6.00 & $\begin{array}{l}\text { Reversibly } \\
\text { compressible }\end{array}$ & Yes & Yes & Yes & $70 \pm 3$ \\
\hline 12.00 & $\begin{array}{l}\text { Reversibly } \\
\text { compressible }\end{array}$ & No & Yes & Yes & $80 \pm 8$ \\
\hline 16.00 & Brittle, rigid & No & Yes & Yes & $144 \pm 4$ \\
\hline 20.00 & Brittle, rigid & No & Yes & Yes & $202 \pm 5$ \\
\hline
\end{tabular}

Table S2: Physical properties of carbon spherogels prepared by templating with different polystyrene sphere concentrations

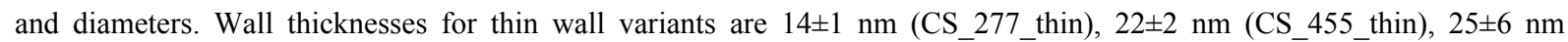

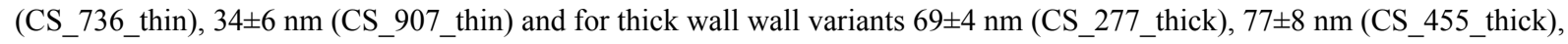
$55 \pm 6 \mathrm{~nm}$ (CS_736_thick), $72 \pm 9 \mathrm{~nm}$ (CS_277_thick).

\begin{tabular}{|c|c|c|c|c|c|c|}
\hline Sample & $\begin{array}{c}\text { SSA } \\
(\text { BET) } \\
\left(\mathrm{m}^{2} / \mathrm{g}\right)\end{array}$ & $\begin{array}{c}\text { Inner } \\
\text { sphere diameter } \\
\text { (TEM) } \\
\text { (nm) }\end{array}$ & $\begin{array}{c}\text { Wall thickness } \\
\text { (TEM) } \\
\text { (nm) }\end{array}$ & $\begin{array}{c}\text { Specific pore } \\
\text { volume } \leq 2 \\
\text { nm } \\
(\mathrm{NLDFT}) \\
\left(\mathrm{cm}^{3} / \mathrm{g}\right)\end{array}$ & $\begin{array}{c}\text { Specific pore } \\
\text { volume } \leq 30 \mathrm{~nm} \\
(\mathrm{NLDFT}) \\
\left(\mathrm{cm}^{3} / \mathrm{g}\right)\end{array}$ & $\begin{array}{c}\text { Bulk density } \\
\left(\mathrm{g} / \mathrm{cm}^{3}\right)\end{array}$ \\
\hline Pristine & $595 \pm 100$ & - & - & 0.21 & 0.35 & $0.060 \pm 0.01$ \\
\hline Pristine_activated & $1652 \pm 100$ & - & - & 0.67 & 0.97 & $0.070 \pm 0.01$ \\
\hline CS_277_thin & $617 \pm 100$ & $196 \pm 7$ & $14 \pm 1$ & 0.23 & 0.44 & $0.053 \pm 0.01$ \\
\hline CS_277_thin_activated & $1822 \pm 100$ & $202 \pm 4$ & $12 \pm 1$ & 0.73 & 1.07 & $0.087 \pm 0.01$ \\
\hline CS_277_thick & $580 \pm 100$ & $201 \pm 3$ & $69 \pm 4$ & 0.24 & 0.34 & $0.051 \pm 0.01$ \\
\hline CS_277_thick_activated & $1931 \pm 100$ & $203 \pm 4$ & $65 \pm 3$ & 0.74 & 1.13 & $0.068 \pm 0.01$ \\
\hline CS_455_thin & $608 \pm 100$ & $334 \pm 22$ & $22 \pm 2$ & 0.24 & 0.49 & $0.064 \pm 0.01$ \\
\hline CS_455_thick & $594 \pm 100$ & $343 \pm 14$ & $77 \pm 8$ & 0.25 & 0.28 & $0.060 \pm 0.01$ \\
\hline CS_736_thin & $629 \pm 100$ & $595 \pm 17$ & $25 \pm 6$ & 0.24 & 0.48 & $0.066 \pm 0.01$ \\
\hline CS_736_thick & $587 \pm 100$ & $613 \pm 22$ & $55 \pm 6$ & 0.25 & 0.32 & $0.055 \pm 0.01$ \\
\hline CS_907_thin & $551 \pm 100$ & $794 \pm 45$ & $34 \pm 6$ & 0.23 & 0.34 & $0.056 \pm 0.01$ \\
\hline CS_907 thick & $699 \pm 100$ & $798 \pm 109$ & $72 \pm 9$ & 0.22 & 0.36 & $0.055 \pm 0.01$ \\
\hline
\end{tabular}

